\title{
MicroRNA-1323 serves as a biomarker in gestational diabetes mellitus and aggravates high glucose-induced inhibition of trophoblast cell viability by suppressing TP53INP1
}

\author{
LIJUN LIU ${ }^{1}$, JUN ZHANG ${ }^{2}$ and YUJUAN LIU ${ }^{3}$ \\ Departments of ${ }^{1}$ Gynecology and ${ }^{2}$ Pharmacy, and ${ }^{3}$ Central Supply Room, \\ Weifang Maternal and Child Health Hospital, Weifang, Shandong 261011, P.R. China
}

Received June 24, 2020; Accepted December 14, 2020

DOI: $10.3892 /$ etm.2021.9661

\begin{abstract}
Gestational diabetes mellitus (GDM) leads to poor pregnancy outcomes, and microRNAs (miRNAs/miRs) have been suggested to be associated with GDM, but the pathological mechanisms remain unclear. The present study aimed to investigate the diagnostic value of miR-1323 in GDM patients and its effects on trophoblast cell viability. Additionally, the present study investigated the correlation between miR-1323 and TP53INP1 to understand the pathological mechanism of GDM progression. Reverse transcription-quantitative polymerase chain reaction was used to detect the miR-1323 expression and TP53INP1 mRNA expression. The diagnostic value of serum miR-1323 was evaluated by receiver operating characteristic analysis. HTR-8/SVneo and BeWo cells were treated with high glucose (HG) to construct cell models of GDM, and trophoblast cell viability was assessed using an MTT assay. The protein expression of TP53INP1 was detected by western blot analysis. The correlation between miR-1323 and TP53INP1 was investigated by luciferase reporter assay. The miR-1323 expression was increased in patients with GDM, which had relatively high diagnostic accuracy for GDM screening and was positively correlated with fasting blood glucose in patients GDM. HG upregulated the miR-1323 expression and inhibited trophoblast cell viability. Overexpression of miR-1323 significantly inhibited the viability of HG-induced trophoblast cells. TP53INP1, a target gene of miR-1323, was negatively correlated with miR-1323. TP53INP1 overexpression reversed the inhibitory effect of miR-1323 overexpression on the viability of HG-treated trophoblast cells. Increased levels of serum miR-1323 may be a diagnostic biomarker for GDM. Additionally, miR-1323
\end{abstract}

Correspondence to: Dr Yujuan Liu, Department of Central Supply Room, Weifang Maternal and Child Health Hospital, 407 Qingnian Road, Weicheng, Weifang, Shandong 261011, P.R. China

E-mail: yujuan0812@163.com

Key words: microRNA-1323, gestational diabetes mellitus, diagnosis, viability, TP53INP1 may inhibit trophoblast cell viability by inhibiting TP53INP1, suggesting that it may be a potential therapeutic target for GDM.

\section{Introduction}

Gestational diabetes mellitus (GDM), the most common metabolic disorder in pregnancy, refers to glucose intolerance occurring in the second and third trimesters of pregnancy, leading to hyperglycemia of varying severity (1). The main drivers of the increased prevalence of GDM include maternal obesity, physical inactivity and increased maternal age (2). GDM increases the risk of severe pregnancy complications for mother and child, including birth trauma, shoulder dystocia, macrosomia, neonatal hypoglycemia and hyperbilirubinemia, and stillbirth (3). The patients with GDM have a significantly increased lifetime risk for type 2 diabetes, while their offspring are more likely to develop type 2 diabetes, obesity, metabolic and cardiovascular disease later in life (4). Therefore, effective prevention and early diagnosis of patients with GDM are very important.

A previous study has reported that maternal diabetes may lead to alterations in placental structure and function, as well as fetal malformation, which has principally been attributed to hyperglycemia (5). The placenta is a highly specific organ for the exchange of gases, nutrients and metabolites between the mother and fetus during pregnancy and is essential for the maintenance of pregnancy (6). Trophoblasts are the first cell lineage to differentiate, invade and migrate to the vascular tissue of the placenta and fetal membrane during pregnancy (7). The inhibition of trophoblast cell viability, invasion and migration of trophoblast cells may contribute toward maldevelopment of placental tissues, which has been reported in certain studies $(8,9)$. Therefore, trophoblast cells with normal biological functions serve crucial roles in placental development and are widely used in the construction of GDM cell models.

MicroRNAs (miRNAs/miRs) are a novel class of small non-coding RNAs, which regulate gene expression via binding to the 3'-untranslated region (3'-UTR) of the target gene (10). miRNAs are important metabolic and developmental regulators during pregnancy and are involved in the development 
of GDM $(11,12)$. Certain studies have reported that miRNAs may serve as potential biomarkers of $\operatorname{GDM}(13,14)$, suggesting that miRNAs may be therapeutic targets of GDM. A previous study reported that the expression of miR-1323 was significantly upregulated in patients with GDM (15). In addition, miR-1323 has been demonstrated to be associated with the placenta and, therefore, may be involved in the regulation of trophoblast function (16). The role of TP53INP1 in metabolic regulation has been reported (17), suggesting that TP53INP1 may regulate GDM. Notably, Zhang et al (18) reported that TP53INP1 was a direct target of miR-1323 and may reverse the effect of miR-1323 on the proliferation of hepatocellular carcinoma cells (18). However, the clinical significance and biological function of miR-1323 in patients with GDM remain unclear. To the best of our knowledge, the interaction between miR-1323 and TP53INP1 has not been revealed in the pathogenesis of GDM to understand the molecular mechanisms.

The present study aimed to assess the expression of miR-1323 in the serum of patients with GDM and trophoblastic cells. Additionally, the present study investigated the diagnostic value of serum miR-1323 in GDM. Furthermore, the effect of miR-1323 on trophoblast viability was analyzed and the correlation between miR-1323 and TP53INP1 was assessed to further understand the underlying molecular mechanisms involved in the biological function of miR-1323.

\section{Materials and methods}

Patients and blood sample collection. The experimental protocols were approved by the Ethics Committee of Weifang Maternal and Child Health Hospital (Weifang, China), and all participants provided written informed consent. The sample size in the present study was determined based on the margin of error (set at 10\%) and confidence level (set at 95\%); therefore, the minimum sample size should be 96 . The present study recruited 110 patients with GDM from Weifang Maternal and Child Health Hospital between March 2014 and May 2018. The patients were diagnosed according to the guidelines of American Diabetes Association (19), and 78 healthy pregnant females were recruited as the control group. The demographic and clinical characteristics of all recruits are summarized in Table I, and there were no significant differences in age, body mass index, gestation and placental weight between the two groups (all $\mathrm{P}>0.05$ ). The average age of patients with GDM was 33.118 \pm 3.402 years (range, 27-42 years) and the average age of the healthy pregnant females was $32.385 \pm 3.579$ years (range, 26-41 years). The patients with pre-gestational diabetes, multiple gestations accompanied with further complications and those taking medications were all excluded from the present study. Blood samples were collected from participants at 24-28 weeks gestational following an overnight fast, and fasting blood glucose (FBG) was measured and recorded by using the glucose oxidase method (20). Additionally, the cut-off value for FBG to diagnose GDM was $5.1 \mathrm{mM} / 1$. Meanwhile, serum was isolated from blood by centrifugation at $1,500 \times \mathrm{g}$ for $10 \mathrm{~min}$ at $4^{\circ} \mathrm{C}$ and stored at $-80^{\circ} \mathrm{C}$ for further use.

Cell culture and treatment. Human trophoblast HTR-8/SVneo and BeWo cell lines were purchased from Cell Bank of
Type Culture Collection of Chinese Academy of Sciences. HTR-8/SVneo cells were cultured in Dulbecco's modified Eagle's medium (DMEM; including 1,000 mg/l glucose; Invitrogen; Thermo Fisher Scientific, Inc.) supplemented with $10 \%$ fetal bovine serum (FBS; Gibco; Thermo Fisher Scientific, Inc.), and BeWo cells were cultured in F-12 medium (Gibco; Thermo Fisher Scientific, Inc.), supplemented with 10\% FBS. Both HTR-8/SVneo and BeWo cells were incubated at $37^{\circ} \mathrm{C}$ in a humidified atmosphere containing $5 \% \mathrm{CO}_{2}$. The cells were divided into two groups: The high glucose (HG)-treated group and the normal cell group. Cells in the HG group were cultured in HG medium with $25 \mathrm{mM}$ glucose, while cells in the normal group were incubated in normal medium with $5 \mathrm{mM}$ glucose at $37^{\circ} \mathrm{C}$ for $72 \mathrm{~h}$.

Transfection of HTR-8/SVneo and BeWo cells. HTR-8/SVneo and BeWo cells were cultured at $37^{\circ} \mathrm{C}$ for $24 \mathrm{~h}$. Subsequently, the cell transfection vectors, including $50 \mathrm{nM}$ miR-1323 mimic (5'-UCAAAACUGAGGGGCAUUUUCU-3'), $100 \mathrm{nM}$ miR-1323 inhibitor (5'-AGAAAAUGCCCCUCAGUUUUG A-3'), $50 \mathrm{nM}$ miR-1323 mimic negative control (mimic NC; 5'-UUCUCCGAACGUGUCACGU-3') and $100 \mathrm{nM}$ miR-1323 inhibitor NC (5'-CAGUACUUUUGUGUAGUACAA-3'), which were obtained from Shanghai GenePharma Co., Ltd., were used for cell transfection to regulate the expression of miR-1323 in trophoblast cells. The TP53INP1 overexpression vector pcDNA3.1-TP53INP1 $(50 \mathrm{nM})$ was constructed to promote the expression of TP53INP1 in trophoblast cells, and the empty vector was used as a control for the overexpression vector pcDNA3.1-TP53INP1. The aforementioned vectors were respectively transfected into trophoblast cells using Lipofectamine ${ }^{\circledR} 3000$ reagent (Invitrogen; Thermo Fisher Scientific, Inc.), according to the manufacturer's protocols. Cell transfection was performed at $37^{\circ} \mathrm{C}$ for $6 \mathrm{~h}$, and subsequently the transfection reagent was removed. After $48 \mathrm{~h}$ of transfection, the cells were used for subsequent experiments.

$R N A$ extraction and reverse transcription-quantitative $P C R$ $(R T-q P C R)$. Total RNA, including miRNA, was extracted from serum samples and cells using TRIzol ${ }^{\circledR}$ reagent (Invitrogen; Thermo Fisher Scientific, Inc.). The purity and concentration of the RNA was evaluated using a NanoDrop 2000 (Thermo Fisher, Scientific, Inc.). Single-strand cDNA was synthesized using the obtained RNA and RT-primer (5'-CTC AACTGGTGTCGTGGAGTCGGCAATTCAGTTGAGAGA AAATG-3') using a PrimeScript RT reagent kit (Takara Biotechnology Co., Ltd.) and stored at $-20^{\circ} \mathrm{C}$. The conditions for cDNA synthesis were as follows: $42^{\circ} \mathrm{C}$ for $30 \mathrm{~min}$ and $85^{\circ} \mathrm{C}$ for $5 \mathrm{sec}$.

The miR-1323 expression in serum and cells, and mRNA expression of the TP53INP1 gene in cells were examined using RT-qPCR, which was performed using a SYBR Green I Master Mix kit (Invitrogen; Thermo Fisher Scientific, Inc.) and a 7300 Real-Time PCR system (Applied Biosystems; Thermo Fisher Scientific, Inc.). All the procedures were performed according to the manufacturer's protocols. The following thermocycling conditions were used for the qPCR: $95^{\circ} \mathrm{C}$ for $10 \mathrm{~min}, 40$ cycles of $95^{\circ} \mathrm{C}$ for $30 \mathrm{sec}, 60^{\circ} \mathrm{C}$ for $15 \mathrm{sec}$ and $72^{\circ} \mathrm{C}$ for $15 \mathrm{sec}$. The miR-1323 and TP53INP1 expression levels were normalized to U6 and $\beta$-actin, respectively. The 
Table I. Clinical characteristics of the females included in the present study.

\begin{tabular}{lccc}
\hline Parameter & Control $(\mathrm{n}=78)$ & GDM $(\mathrm{n}=110)$ & P-value \\
\hline Age, years & $32.385 \pm 3.579$ & $33.118 \pm 3.402$ & 0.156 \\
BMI, kg/m ${ }^{2}$ & $22.587 \pm 3.614$ & $23.466 \pm 3.326$ & 0.087 \\
Pregnancy weeks, weeks & $25.218 \pm 2.196$ & $25.591 \pm 1.757$ & 0.198 \\
Placental weight, kg & $0.586 \pm 0.088$ & $0.616 \pm 0.155$ & 0.128
\end{tabular}

GDM, gestational diabetes mellitus; BMI, body mass index.

primer sequences were as follows: miR-1323 forward, 5'-GCC GAGUCAAAACUGAGG-3' and reverse, 5'-CTCAACTGG TGTCGTGGA-3'; U6 forward, 5'-CTCGCTTCGGCAGCA CA-3' and reverse, 5'-AACGCTTCACGAATTTGCGT-3'; TP53INP1 forward, 5'-GCACCCTTCAGTCTTTTCCTG TT-3' and reverse, 5'-GGAGAAAGCAGGAATCACTTGTAT C-3'; $\beta$-actin forward, 5'-CTGGGACGACATGGAGAAAA-3' and reverse, 5'-AAGGAAGGCTGGAAGAGTGC-3'. Each value was calculated using the $2^{-\Delta \Delta \mathrm{Cq}}$ method (21).

MTT assay. The viability of HTR-8/SVneo and BeWo cells was determined by MTT assay. Cells were seeded onto 96-well microplates at a density of $3 \times 10^{4}$ cells/well and incubated at $37^{\circ} \mathrm{C}$ for $24 \mathrm{~h}$. At the time points of $0,24,48$ and $72 \mathrm{~h}$, MTT $(0.5 \mathrm{mg} / \mathrm{ml})$ was added into the wells and was further incubated for $4 \mathrm{~h}$. Next, the supernatant was removed and $200 \mu 1$ dimethyl sulfoxide was added into the wells. The cell viability was examined by reading the absorbance at $490 \mathrm{~nm}$.

Western blot analysis. The cells were lysed using RIPA lysis buffer (Thermo Fisher Scientific, Inc.) to obtain total proteins. The BCA method was used to determine the concentration of proteins. Following quantitation with a WBC Protein Quantitation kit (Thermo Fisher Scientific, Inc.), total proteins were separated via SDS-PAGE (12\% gel) and transferred onto PVDF membranes (EMD Millipore). After $4 \mathrm{~h}$ of blocking with 5\% skimmed milk at $4^{\circ} \mathrm{C}$, the primary antibodies, including anti-TP53INP1 (1:500; cat. no. A04229; Wuhan Boster Biological Technology, Ltd.) and anti- $\beta$-actin (dilution, 1:500; cat. no. BA2305; B Wuhan Boster Biological Technology, Ltd.), were incubated with the membranes at $4^{\circ} \mathrm{C}$ overnight. Next, the membranes were incubated with an HRP-conjugated secondary antibody (1:5,000; cat. no. BA1054; Wuhan Boster Biological Technology, Ltd.) at $37^{\circ} \mathrm{C}$ for $2 \mathrm{~h}$. In this analysis, $\beta$-actin was used as an internal control. Subsequently, an enhanced chemiluminescence BeyoECL Plus kit (Beyotime Institute of Biotechnology) was applied for protein band visualization. The protein bands were quantified using ImageJ Software v1.46 (National Institutes of Health).

Luciferase reporter assay. According to the TargetScan's bioinformatics prediction (version 7.2; www.targetscan. org/vert_72), complementary sequences of miR-1323 were searched at the 3'-UTR of TP53INP1. To confirm the interaction between miR-1323 and TP53INP1, a luciferase reporter assay was performed. The wild-type (WT) 3'-UTR containing the binding site of miR-1323 or mutant-type (MT) 3'-UTR were inserted into the pGL-control vector (Promega).
According to the manufacturer's protocols, the combined vectors were co-transfected into HTR-8/SVneo and BeWo cells with miR-1323 mimic, miR-1323 inhibitor or the NCs using Lipofectamine 3000 reagent. After $48 \mathrm{~h}$ of transfection, relative luciferase activity was measured using a Dual-Luciferase Reporter assay system (Promega Corporation) and normalized to Renilla luciferase activity.

Statistical analysis. All statistical analyses were performed using SPSS 21.0 software (IBM Corp.) and GraphPad Prism 7.0 software (GraphPad Software, Inc.). All data are presented as the mean \pm standard deviation. Data between the two groups were compared using unpaired Student's t-test. One-way analysis of variance followed by Tukey's test was used to analyze the differences between multiple groups. Pearson's correlation analysis was performed to assess the correlation between indicators. Receiver operating characteristic (ROC) curves were plotted to assess the diagnostic value of miR-1323 in patients with GDM. $\mathrm{P}<0.05$ was considered to indicate a statistically significant difference.

\section{Results}

Diagnostic value of upregulated serum miR-1323 and its correlation with FBG in patients with GDM. As shown in Fig. 1A, a significant increase in serum expression of miR-1323 was observed in patients with GDM, compared with that in the healthy controls $(\mathrm{P}<0.05)$. Increased blood glucose is a characteristic of GDM, and Fig. 1B shows a positive correlation between miR-1323 and FBG $(\mathrm{r}=0.721 ; \mathrm{P}<0.001)$. In addition, in patients with GDM the FBG level was $6.532 \pm 0.344 \mathrm{mM} / 1$ and the coefficient of variation was $5.267 \%$. The ROC curve indicated that miR-1323 had high diagnostic accuracy with an AUC of 0.917 . At an optimal cut-off value of 1.489 , the sensitivity was $77.27 \%$ and the specificity was $94.87 \%$ (Fig. 1C).

Effect of HG on miR-1323 expression and trophoblast viability in HTR-8/SVneo and BeWo cells. Following HG treatment, the expression of miR-1323 was upregulated in HG-treated cells, compared with that in normal cells in HTR-8/SVneo and BeWo cell lines (all $\mathrm{P}<0.05$; Fig. $2 \mathrm{~A}$ and $\mathrm{B}$ ). In HTR-8/SVneo and BeWo cells, the trophoblast viability was significantly downregulated in HG-treated cells compared with that in normal cells at 48 and $72 \mathrm{~h}$ (all $\mathrm{P}<0.05$; Fig. $2 \mathrm{C}$ and D).

Effect of miR-1323 on trophoblast viability in HG-treated HTR-8/SVneo and BeWo cells. Following cell transfection, 

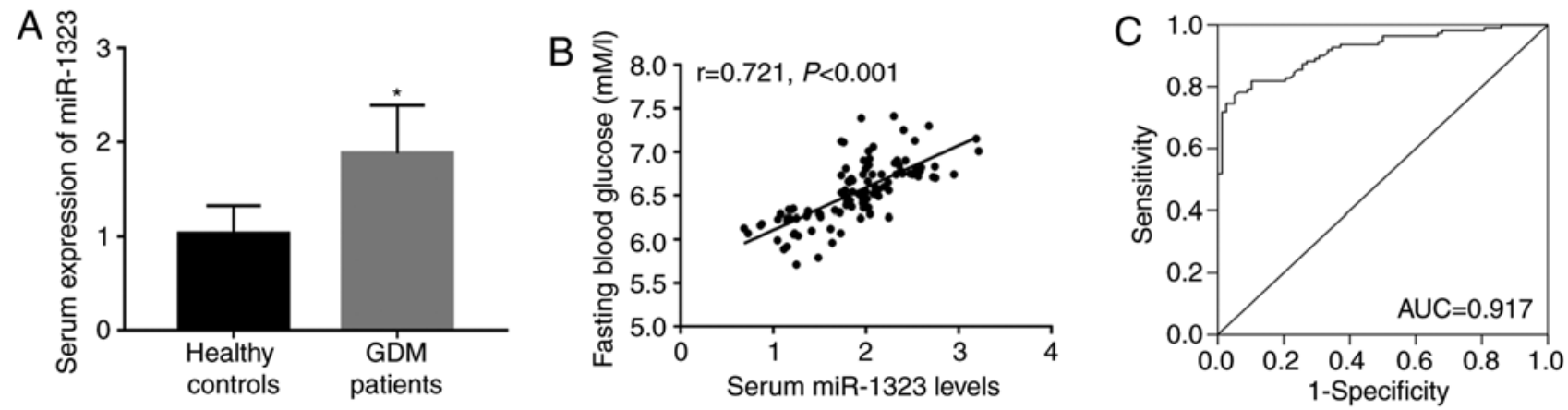

Figure 1. Diagnostic value of upregulated expression of miR-1323 and its correlation with FBG in patients with GDM. (A) Expression of miR-1323 in serum was upregulated in GDM patients, compared with the healthy controls. (B) Serum miR-1323 levels were positively correlated with the FBG of patients with GDM ( $\mathrm{r}=0.721$; $\mathrm{P}<0.001)$. (C) Receiver operating characteristic curve based on serum miR-1323 expression for GDM was plotted. The AUC was 0.917, suggesting the relatively high diagnostic accuracy for serum miR-1323 expression. " $\mathrm{P}<0.05$ vs. healthy controls. miR, microRNA; FBG, fasting blood glucose; GDM, gestational diabetes mellitus; AUC, area under the curve.

A

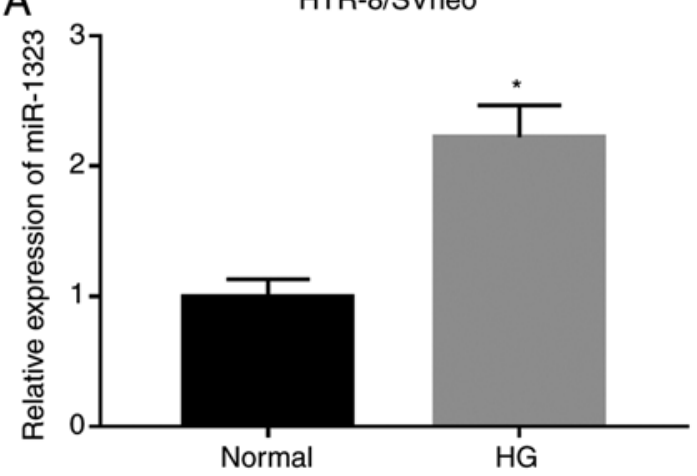

C

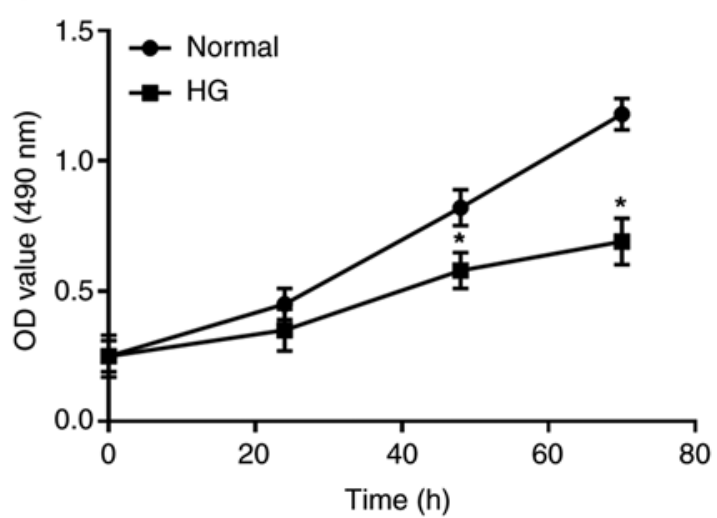

B

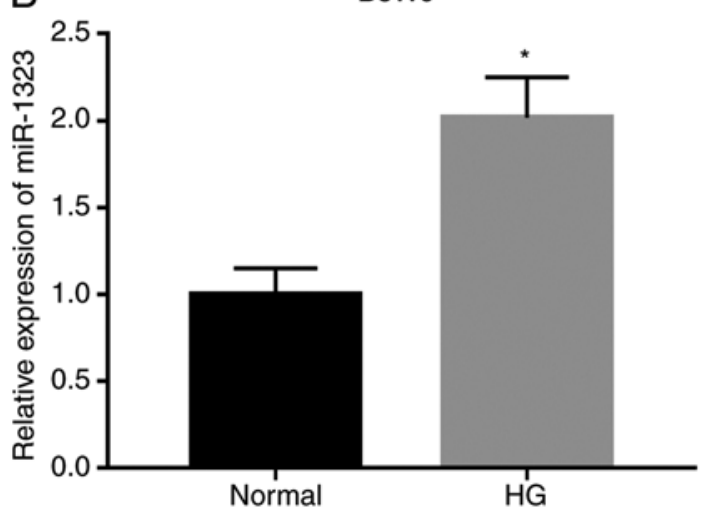

BeWo

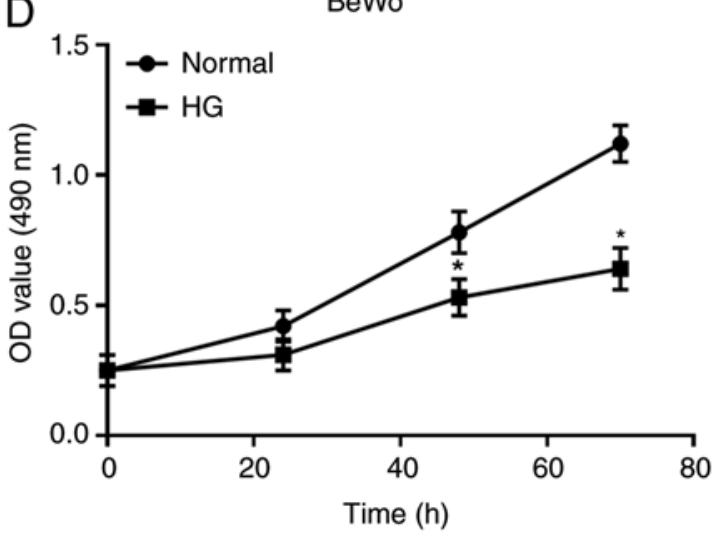

Figure 2. Effect of HG on miR-1323 expression and trophoblast viability in HTR-8/SVneo and BeWo cells. (A and B) Expression of miR-1323 was upregulated in HG-treated cells, compared with that in normal controls in the two cell lines. (C and D) HG suppressed the trophoblast cell viability in the two cell lines. ${ }^{*} \mathrm{P}<0.05$ vs. normal control. HG, high glucose; miR, microRNA.

the expression of miR-1323 was significantly upregulated by the miR-1323 mimic, but was significantly downregulated by the miR-1323 inhibitor in HG-treated HTR-8/SVneo and BeWo cells (all $\mathrm{P}<0.05$; Fig. $3 \mathrm{~A}$ and $\mathrm{B}$ ). The analyses of trophoblast viability suggested that the cell viability was markedly suppressed by the miR-1323 overexpression, but was significantly promoted by miR-1323-knockdown at 48 and $72 \mathrm{~h}$ in both HTR-8/SVneo and BeWo cells (all $\mathrm{P}<0.05$; Fig. 3C and D).
miR-1323 directly regulates TP53INP1 expression in HTR-8/SVneo and BeWo cells. In order to further confirm the association of miR-1323 with TP53INP1, a luciferase reporter assay was performed. The putative binding site of miR-1323 at the 3'-UTR of TP53INP1 was identified (Fig. 4A). In the WT group, the relative luciferase activity was inhibited by the overexpression of miR-1323, but was promoted by the knockdown of miR-1323 in HTR-8/SVneo and BeWo cells (Fig. 4B). However, there were no changes in 

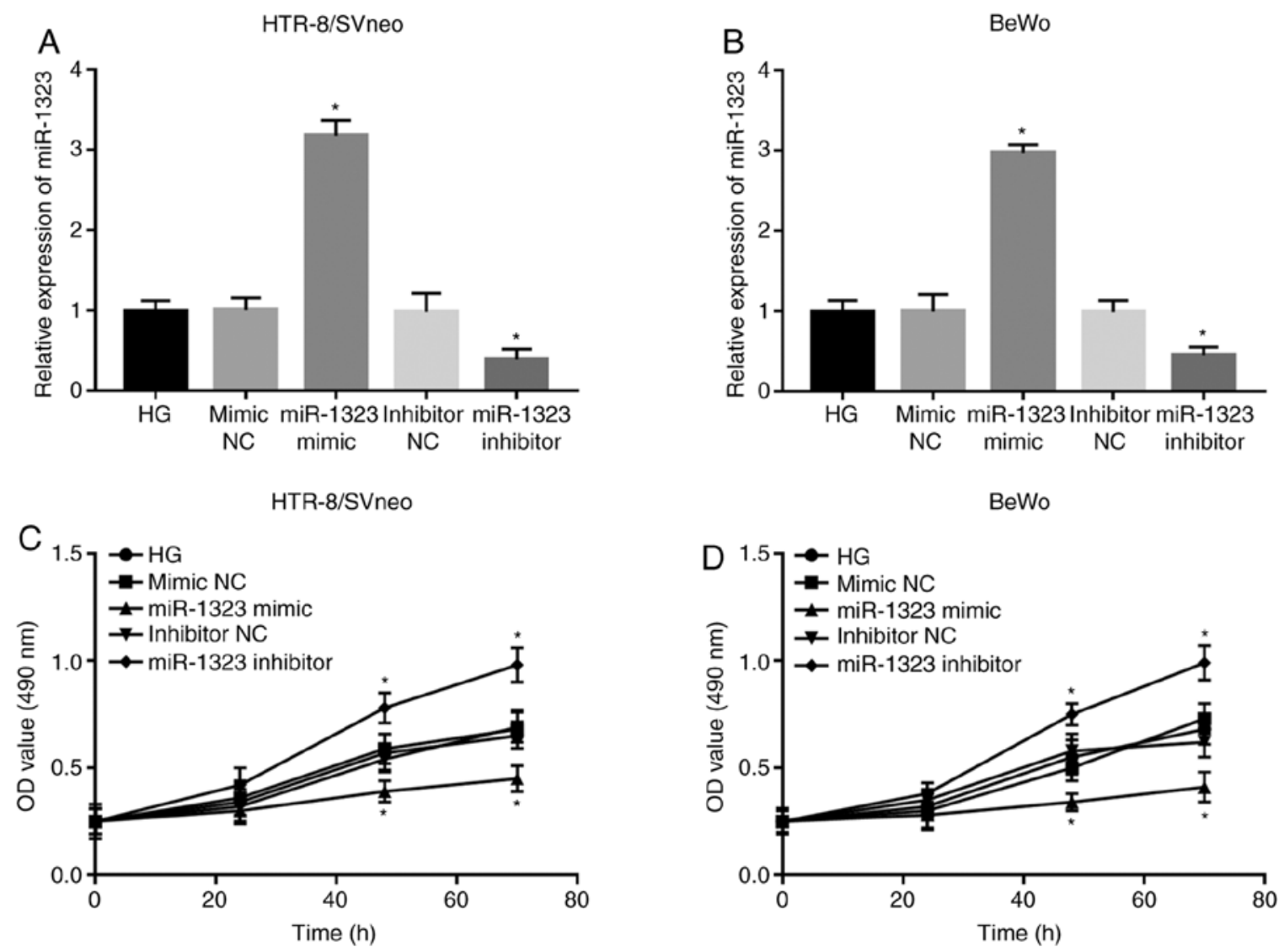

Figure 3. Effect of miR-1323 on trophoblast cell viability in HG-treated HTR-8/SVneo and BeWo cells. (A and B) miR-1323 expression was upregulated by miR-1323 mimic, but was downregulated by miR-1323 inhibitor in two HG-treated trophoblast cell lines. (C and D) Upregulation of miR-1323 suppressed cell viability, while downregulation of miR-1323 promoted cell viability in two HG-treated trophoblast cell lines. "P<0.05 vs. HG-treated group. miR, microRNA; HG, high glucose.

the MUT group in HTR-8/SVneo and BeWo cells (Fig. 4C). Additionally, Fig. 4D and E demonstrate that the overexpression of miR-1323 inhibited, while the knockdown of miR-1323 promoted, the TP53INP1 mRNA expression in HTR-8/SVneo and BeWo cells (all $\mathrm{P}<0.05$ ). Furthermore, the relative protein expression of TP53INP1 in two cell lines was detected, and it was revealed that the protein expression of TP53INP1 was inhibited by overexpression of miR-1323 and was promoted by the knockdown of miR-1323 (all P<0.05; Fig. 4F and G).

Effect of miR-1323/TP53INP1 on trophoblast viability in HG-treated HTR-8/SVneo and BeWo cells. The expression of TP53INP1 was suppressed by HG treatment, and was promoted by the pcDNA3.1-TP53INP1 in HG-treated trophoblast cells. Additionally, the expression of TP53INP1, which was suppressed by miR-1323 overexpression, was promoted by pcDNA3.1TP53INP1 in HG-treated HTR-8/SVneo and BeWo cells (all $\mathrm{P}<0.05$; Fig. $5 \mathrm{~A}$ and $\mathrm{B}$ ). The inhibition of trophoblast viability because of miR-1323 overexpression was reversed by the overexpression of TP53INP1 (all P<0.05; Fig. 5C and D). These data suggested that miR-1323 may inhibit the trophoblast cell viability by suppressing TP53INP1 in HTR-8/SVneo and BeWo cells.

\section{Discussion}

In recent years, microRNAs (miRNAs) have been recognized as important molecules in biological processes and are associated with various human diseases (22). For example, Fang et al (23) reported that miR-185 promoted the progression of atherosclerosis by targeting STIM1. A study by Liu et al (24) demonstrated that miR-34a promoted renal fibrosis by downregulating Klotho in tubular epithelial cells. Certain miRNAs with abnormal expression have been reported in GDM, including miR-132 (25) and miR-185 (26). In addition, it has been reported that miR-1323 is upregulated in patients with GDM (15) and may be involved in the regulation of trophoblast function (16). These studies highlighted the roles of miRNAs in GDM. The present study also identified that serum miR-1323 expression was upregulated in patients with GDM and was positively correlated with FBG. Therefore, we hypothesized that miR-1323 may be involved in GDM.

miRNAs have been considered as ideal diagnostic tools for various human diseases due to their abnormal expression and stability in blood samples (27). For example, decreased levels of serum miR-103 may be used as non-invasive diagnostic biomarkers in patients with sepsis (28) and decreased levels of serum miR-375 may serve as biomarkers for the diagnosis of osteosarcoma (29). Considering the increased expression of miR-1323 in patients with GDM, ROC curves were plotted based on the miR-1323 expression and revealed that miR-1323 may be a potential diagnostic biomarker for patients with GDM. Abnormal serum miR-1323 expression has been reported to be a candidate biomarker for numerous other diseases, including lung adenocarcinoma (30) and breast 


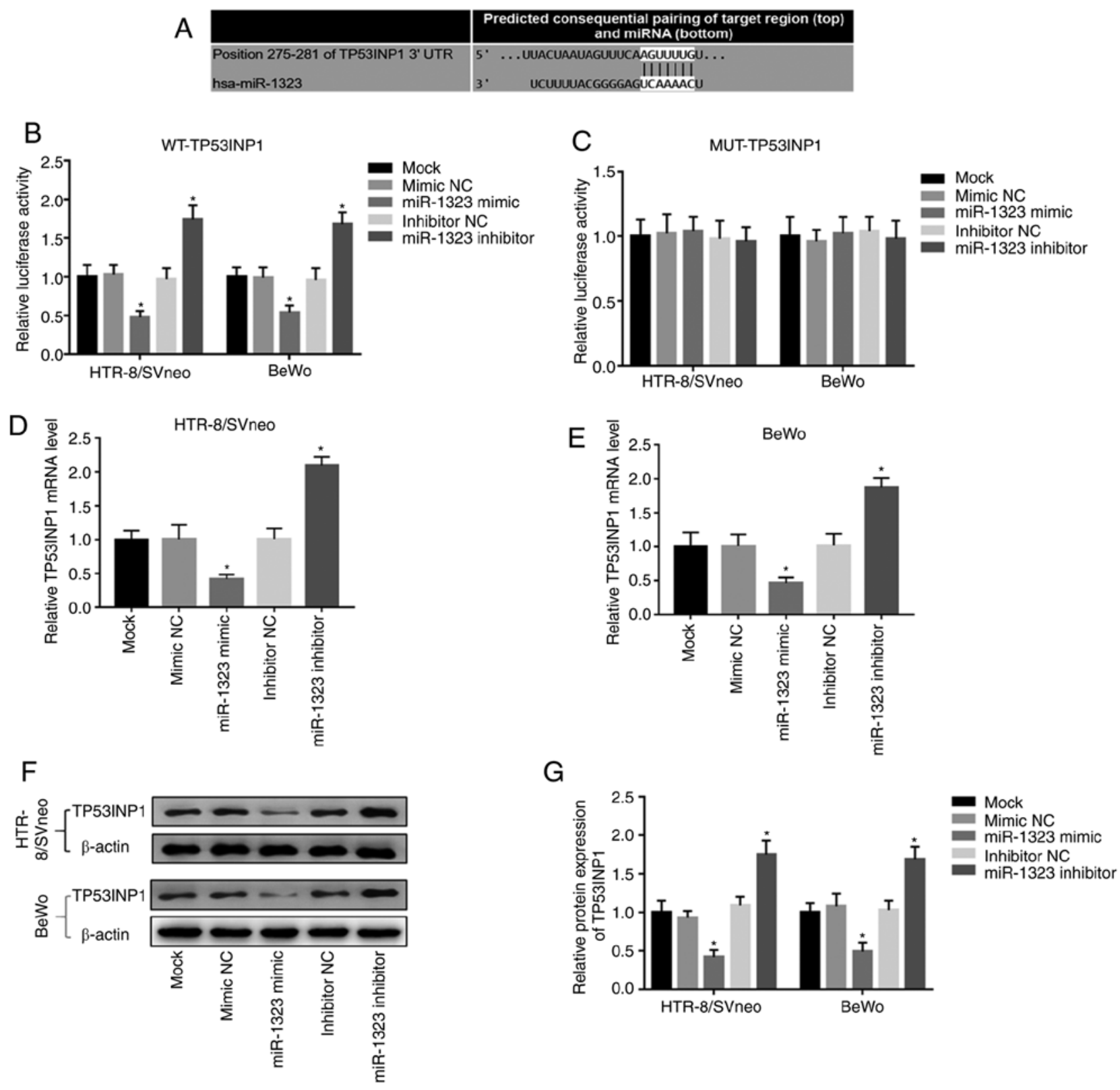

Figure 4. miR-1323 directly regulates TP53INP1 level in HTR-8/SVneo and BeWo cells. (A) A putative binding site of miR-1323 at the 3'-UTR of TP53INP1. (B) In two cell lines, luciferase activity was inhibited by miR-1323 overexpression, but was promoted by miR-1323 knockdown in the WT group. (C) No changes were observed in the luciferase activity in MUT group. (D and E) The TP53INP1 level was inhibited by miR-1323-overexpression, but was promoted by miR-1323-knockdown in two cell lines. (F and G) Western blot analysis and quantification of TP53INP1 proteins in two cell lines. ${ }^{*} \mathrm{P}<0.05$. miR, microRNA; UTR, untranslated region; WT, wild-type; MUT, mutant.

cancer (31). Therefore, we hypothesized that serum miR-1323 may be a novel and effective diagnostic biomarker for patients with GDM.

It is well known that the normal growth and development of the placenta is one of the important steps of a healthy pregnancy. However, patient with GDM often have placental dysplasia (5). HG is a characteristic of GDM, resulting in impaired trophoblast function, thereby inhibiting normal development of the placenta (32). Therefore, HG-induced trophoblast cells are widely used to study the molecules involved in the biological function of trophoblast cells during the pathological process of GDM. In the present study, HG markedly inhibited the viability of HTR-8/SVneo and BeWo cells, which is consistent with the results of a previous study (25).
Previous studies have demonstrated that miRNAs may regulate the viability or proliferation of trophoblasts in diseases, including GDM. For example, upregulation of miR-211 repressed the proliferation of JEG-3 and BeWo cells in choriocarcinoma (33). Peng et al (34) reported that miR-137 suppressed the viability of HTR-8/SVneo cells in patients with GDM. In the present study, miR-1323 was upregulated in HG-induced trophoblast cells, compared with the normal trophoblast cells. Additionally, miR-1323 overexpression inhibited, and miR-1323 knockdown promoted the viability of HTR-8/SVneo and BeWo cells in the HG-treated cells. Additionally, previous studies have demonstrated that miR-1323 may affect disease by regulating cell viability or proliferation. For example, suppression of miR-1323 has 

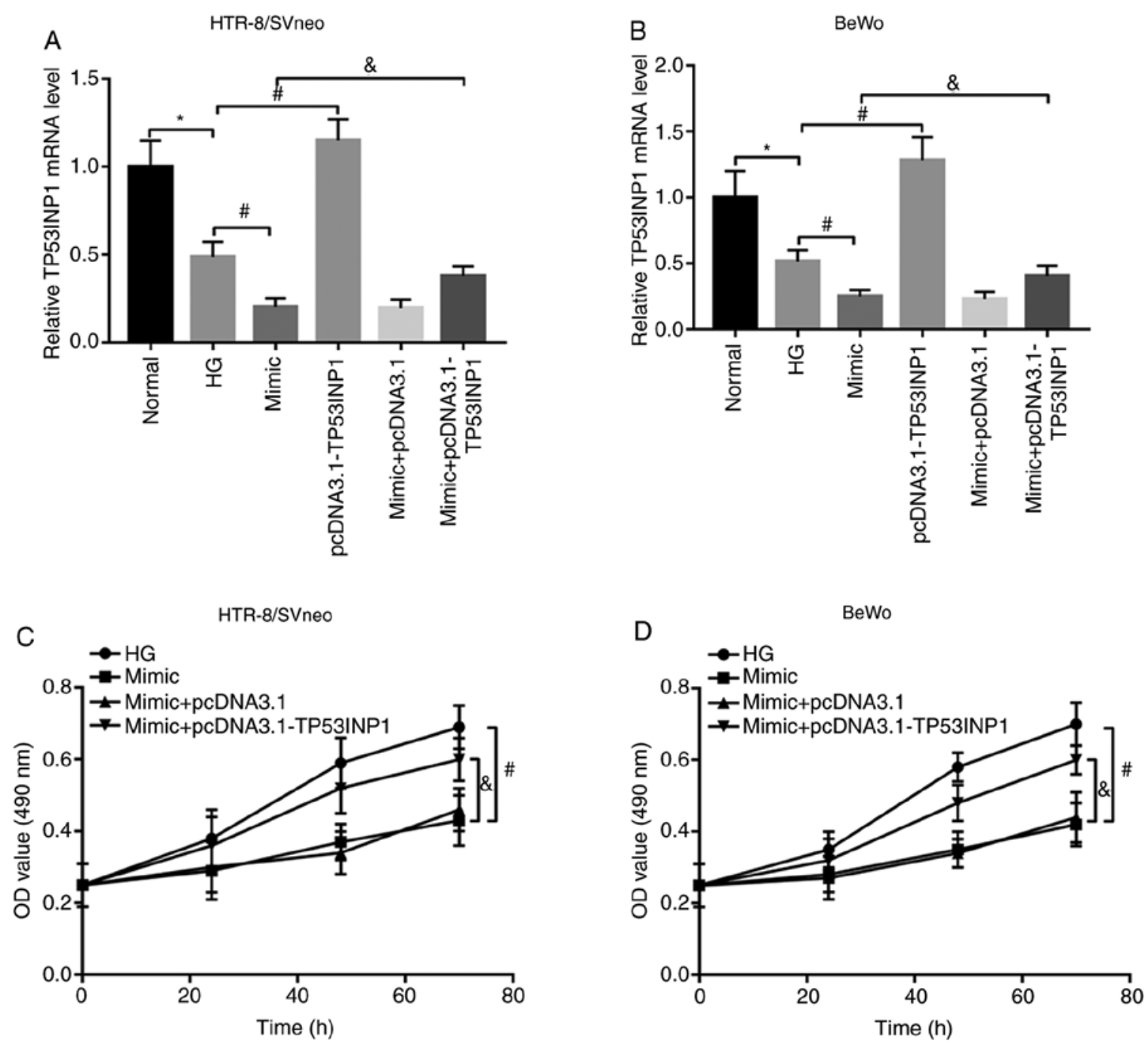

Figure 5. TP53INP1 reverses the inhibitory effect of miR-1323 on trophoblast viability in HG-treated HTR-8/SVneo and BeWo cells. (A and B) TP53INP1 expression was inhibited by HG treatment in the two cell lines. In the HG-treated two cell lines, the inhibited TP53INP1 expression by miR-1323 overexpression was increased by the pcDNA3.1-TP53INP1. (C and D) In the HG-treated two cell lines, the increase in TP53INP1 expression reversed the inhibitory effect of miR-1323 overexpression on trophoblast cell viability. ${ }^{*} \mathrm{P}<0.05$ vs. normal; ${ }^{*} \mathrm{P}<0.05$ vs. HG; and ${ }^{\circledR} \mathrm{P}<0.05$ vs. mimic. miR, microRNA; HG, high glucose.

been reported to promote breast cancer cell viability (31). In addition, a study by Gillet et al (15) reviewed the literature and reported that miR-1323 was involved in trophoblast proliferation in pregnant females. Therefore, we hypothesized that miR-1323 may exert a cytotoxic role in GDM by inhibiting trophoblast cell viability.

TP53INP1, a key stress protein with antioxidant-associated tumor suppressor function, is involved in cell cycle arrest and apoptosis (35). Numerous studies $(18,36,37)$ have demonstrated that TP53INP1 may serve as a target of miRNA and was regulated by miRNA in diseases. For example, Du et al (36) reported that TP53INP1 was a target of miR-142-5p and was inhibited by miR-142-5p in heart failure caused by myocardial infarction. Li et al (37) found that TP53INP1 was a target of miR-155-5p and regulated by miR-155-5p in cervical cancer. Notably, a study by Zhang et al (18) suggested that TP53INP1 was a direct target of miR-1323 and was inhibited by miR-1323 in hepatocellular carcinoma. Therefore, the present study focused on the effect of miR-1323 on the levels of TP53INP1 in patients with GDM. The result suggested that TP53INP1 was the target of miR-1323, the mRNA and protein levels of TP53INP1 were downregulated by miR-1323-overexpression. In addition, it has been reported that miRNAs regulate cell viability by regulating TP53INP1 in other diseases. For example, Ye et al (38) reported that miR-3934-5p may inhibit the cell viability of neuroblastoma by targeting TP53INP1. TP53INP1 expression was negatively regulated by miR-301a, and TP53INP1/miR-301a was involved in gastric cancer cell viability (39). Zhang et al (18) reported that miR-1323 promoted the proliferation of hepatocellular carcinoma cells by targeting the TP53INP1. In the present study, in trophoblasts co-transfected with miR-1323 and TP53INP1 overexpression vectors, in was revealed that the cell viability inhibited by miR-1323 overexpression was reversed by TP53INP1 overexpression. These results suggested that the inhibition of trophoblast viability by miR-1323 may be achieved by inhibiting TP53INP1. miR-1323 may be a potential therapeutic target for GDM treatment.

In conclusion, the results of the present study suggested that the upregulated level of serum miR-1323 may serve as a diagnostic biomarker for GDM. In addition, knockdown of miR-1323 in trophoblast cells may promote the trophoblast 
cell viability under HG conditions by targeting TP53INP1, suggesting that miR-1323 may be a potential therapeutic target for GDM treatment. However, only in vitro experiments were conducted in the present study, and further in vivo experiments are required to confirm the role of the miR-1323/TP53INP1 axis in the pathogenesis of GDM. Another limitation was identified in the present study. A recent study has reported that the HTR-8/SVneo cell line contains two populations, one of epithelial and one of mesenchymal origin (40). However, this was not taken into consideration in the current study, which is a limitation. Therefore, further studies need to focus on the different populations in the HTR-8/SVneo cells, and further conclusions may emerge.

\section{Acknowledgements}

Not applicable.

\section{Funding}

No funding was received.

\section{Availability of data and materials}

The datasets used and/or analyzed during the current study are available from the corresponding author on reasonable request.

\section{Authors' contributions}

LL and YL designed and conceived the study, conducted the clinical studies, analyzed the clinical data, wrote the manuscript and confirmed the authenticity of the raw data. JZ performed the cell experiments and analyzed the corresponding data. All authors read and approved the final manuscript.

\section{Ethics approval and consent to participate}

Written informed consent was obtained from each patient and the experimental procedures were approved by the Ethics Committee of Weifang Maternal and Child Health Hospital (Weifang, China).

\section{Patient consent for publication}

Written informed consent for publication was obtained from each participant.

\section{Competing interests}

The authors declare that they have no competing interests.

\section{References}

1. Chiefari E, Arcidiacono B, Foti D and Brunetti A: Gestational diabetes mellitus: An updated overview. J Endocrinol Invest 40: 899-909, 2017.

2. Lavery JA, Friedman AM, Keyes KM, Wright JD and Ananth CV: Gestational diabetes in the United States: Temporal changes in prevalence rates between 1979 and 2010. BJOG 124: 804-813, 2017.

3. Committee on Practice Bulletins-Obstetrics: ACOG Practice Bulletin No. 190: Gestational diabetes mellitus. Obstet Gynecol 131: e49-e64, 2018.
4. Yung HW, Alnaes-Katjavivi P, Jones CJ, El-Bacha T, Golic M, Staff AC and Burton GJ: Placental endoplasmic reticulum stress in gestational diabetes: The potential for therapeutic intervention with chemical chaperones and antioxidants. Diabetologia 59: 2240-2250, 2016

5. Edu A, Teodorescu C, Dobjanschi CG, Socol ZZ, Teodorescu V, Matei A, Albu DF and Radulian G: Placenta changes in pregnancy with gestational diabetes. Rom J Morphol Embryol 57: 507-512, 2016.

6. Wang Y, Ji L, Peng Z, Lai R, Zhang X, Xu Y, Chen Z, Liu R, Zhong $\mathrm{Y}, \mathrm{Hu} \mathrm{H}$ and Wang L: Silencing DAPK3 blocks the autophagosome-lysosome fusion by mediating SNAP29 in trophoblast cells under high glucose treatment. Mol Cell Endocrinol 502: 110674, 2020.

7. Chang SC and Vivian Yang WC: Hyperglycemia induces altered expressions of angiogenesis associated molecules in the trophoblast. Evid Based Complement Alternat Med 2013: 457971, 2013

8. Zong S, Li C, Luo C, Zhao X, Liu C, Wang K, Jia W, Bai M, Yin M, Bao S, et al: Dysregulated expression of IDO may cause unexplained recurrent spontaneous abortion through suppression of trophoblast cell proliferation and migration. Sci Rep 6: 19916, 2016.

9. Tian FJ, Qin CM, Li XC, Wu F, Liu XR, Xu WM and Lin Y: Decreased stathmin-1 expression inhibits trophoblast proliferation and invasion and is associated with recurrent miscarriage. Am J Pathol 185: 2709-2721, 2015.

10. Gao Y, She R, Wang Q, Li Y and Zhang H: Up-regulation of miR-299 suppressed the invasion and migration of HTR-8/SVneo trophoblast cells partly via targeting HDAC2 in pre-eclampsia. Biomed Pharmacother 97: 1222-1228, 2018.

11. Dias S, Pheiffer C, Abrahams Y, Rheeder P and Adam S: Molecular biomarkers for gestational diabetes mellitus. Int J Mol Sci 19: 2926, 2018

12. Tryggestad JB, Vishwanath A, Jiang S, Mallappa A, Teague AM, Takahashi Y, Thompson DM and Chernausek SD: Influence of gestational diabetes mellitus on human umbilical vein endothelial cell miRNA. Clin Sci (Lond) 130: 1955-1967, 2016.

13. Pheiffer C, Dias S, Rheeder P and Adam S: Decreased expression of circulating miR-20a-5p in South African women with gestational diabetes mellitus. Mol Diagn Ther 22: 345-352, 2018.

14. Yoffe L, Polsky A, Gilam A, Raff C, Mecacci F, Ognibene A, Crispi F, Gratacós E, Kanety H, Mazaki-Tovi S, et al: Early diagnosis of gestational diabetes mellitus using circulating microRNAs. Eur J Endocrinol 181: 565-577, 2019.

15. Gillet V, Ouellet A, Stepanov Y, Rodosthenous RS, Croft EK, Brennan K, Abdelouahab N, Baccarelli A and Takser L: miRNA profiles in extracellular vesicles from serum early in pregnancies complicated by gestational diabetes mellitus. J Clin Endocrinol Metab 104: 5157-5169, 2019.

16. Na Q, Wang D and Song W: Underexpression of 4 placenta-associated microRNAs in complete hydatidiform moles. Int J Gynecol Cancer 22: 1075-1080, 2012.

17. Seillier M, Pouyet L, N'Guessan P, Nollet M, Capo F, Guillaumond F, Peyta L, Dumas JF, Varrault A, Bertrand G, et al: Defects in mitophagy promote redox-driven metabolic syndrome in the absence of TP53INP1. EMBO Mol Med 7: 802-818, 2015.

18. Zhang F, Yang C, Xing Z, Liu P, Zhang B, Ma X, Huang L and Zhuang L: LncRNA GAS5-mediated miR-1323 promotes tumor progression by targeting TP53INP1 in hepatocellular carcinoma. Onco Targets Ther 12: 4013-4023, 2019.

19. American Diabetes Association: Diagnosis and classification of diabetes mellitus. Diabet Care 35 (Suppl 1): S64-S71, 2012.

20. Tiongco RE, Bituin A, Arceo E, Rivera N and Singian E: Salivary glucose as a non-invasive biomarker of type 2 diabetes mellitus. J Clin Exp Dent 10: e902-e907, 2018.

21. Livak KJ and Schmittgen TD: Analysis of relative gene expression data using real-time quantitative PCR and the 2(-Delta Delta C(T)) method. Methods 25: 402-408, 2001.

22. Chen X, Gong Y,Zhang DH, You ZH and Li ZW: DRMDA: Deep representations-based miRNA-disease association prediction. J Cell Mol Med 22: 472-485, 2018.

23. Fang M, Li Y, Wu Y, Ning Z, Wang X and Li X: miR-185 silencing promotes the progression of atherosclerosis via targeting stromal interaction molecule 1. Cell Cycle 18: 682-695, 2019.

24. Liu Y, Bi X, Xiong J, Han W, Xiao T, Xu X, Yang K, Liu C, Jiang W, He T, et al: MicroRNA-34a promotes renal fibrosis by downregulation of klotho in tubular epithelial cells. Mol Ther 27: 1051-1065, 2019. 
25. Zhou $X$, Xiang $C$ and Zheng $X$ : miR-132 serves as a diagnostic biomarker in gestational diabetes mellitus and its regulatory effect on trophoblast cell viability. Diagn Pathol 14: 119, 2019.

26. Qi S and Wang X: Decreased expression of miR-185 in serum and placenta of patients with gestational diabetes mellitus. Clin Lab 65, 2019.

27. Bertoli G, Cava C and Castiglioni I: The potential of miRNAs for diagnosis, treatment and monitoring of breast cancer. Scand J Clin Lab Invest Suppl 245 (Suppl 1): S34-S39, 2016.

28. Yang M, Zhao L and Sun M: Diagnostic value of miR-103 in patients with sepsis and noninfectious SIRS and its regulatory role in LPS-induced inflammatory response by targeting TLR4. Int J Genomics 2020: 2198308, 2020.

29. Liu W, Zhao X, Zhang YJ, Fang GW and Xue Y: MicroRNA-375 as a potential serum biomarker for the diagnosis, prognosis, and chemosensitivity prediction of osteosarcoma. J Int Med Res 46: 975-983, 2018.

30. Zhao H, Zheng C, Wang Y, Hou K, Yang X, Cheng Y, Che X, Xie S, Wang S, Zhang T, et al: miR-1323 promotes cell migration in lung adenocarcinoma by targeting Cbl-b and is an early prognostic biomarker. Front Oncol 10: 181, 2020.

31. Xu Y and Liu M: MicroRNA-1323 downregulation promotes migration and invasion of breast cancer cells by targeting tumor protein D52. J Biochem 168: 83-91, 2020.

32. Peng HY, Li MQ and Li HP: High glucose suppresses the viability and proliferation of HTR8/SVneo cells through regulation of the miR137/PRKAA1/IL6 axis. Int J Mol Med 42: 799-810, 2018.

33. Ji L and $\mathrm{Li} \mathrm{X}$ : Long noncoding RNA MEG3 is a tumor suppressor in choriocarcinoma by upregulation of microRNA-211. J Cell Physiol 234: 22911-22920, 2019.
34. Peng HY, Li MQ and Li HP: miR-137 restricts the viability and migration of HTR-8/SVneo cells by downregulating FNDC5 in gestational diabetes mellitus. Curr Mol Med 19: 494-505, 2019.

35. Nishimoto M, Nishikawa S, Kondo N, Wanifuchi-Endo Y, Hato Y, Hisada T, Dong Y, Okuda K, Sugiura H, Kato H, et al: Prognostic impact of TP53INP1 gene expression in estrogen receptor $\alpha$-positive breast cancer patients. Jpn J Clin Oncol 49: 567-575, 2019.

36. Du J, Yang ST, Liu J, Zhang KX and Leng JY: Silence of LncRNA GAS5 protects cardiomyocytes H9c2 against hypoxic injury via sponging miR-142-5p. Mol Cells 42: 397-405, 2019.

37. Li N, Cui T, Guo W, Wang D and Mao L: miR-155-5p accelerates the metastasis of cervical cancer cell via targeting TP53INP1. Onco Targets Ther 12: 3181-3196, 2019.

38. Ye W, Liang F, Ying C, Zhang M, Feng D and Jiang X: Downregulation of microRNA-3934-5p induces apoptosis and inhibits the proliferation of neuroblastoma cells by targeting TP53INP1. Exp Ther Med 18: 3729-3736, 2019.

39. Dou J, Tu D, Zhao H and Zhang X: LncRNA PCAT18/miR301a/TP53INP1 axis is involved in gastric cancer cell viability, migration, and invasion. J Biochem 168: 547-555, 2020.

40. Abou-Kheir W, Barrak J, Hadadeh O and Daoud G: HTR-8/SVneo cell line contains a mixed population of cells. Placenta 50: 1-7, 2017.

(i) $\odot$ This work is licensed under a Creative Commons Attribution-NonCommercial-NoDerivatives 4.0 International (CC BY-NC-ND 4.0) License. 